\title{
A SEARCH FOR INTERSTELLAR GAS-PHASE $\mathrm{CO}_{2}$
}

\author{
E.F. VAN DISHOECK AND F.P. HELMICH \\ Sterrewacht Leiden P.O.-Box 9513 \\ 2300 RA Leiden, The Netherlands
}

\section{Introduction}

$\mathrm{CO}_{2}$ is one of the simplest carbon- and oxygen-containing molecules, for which little is known in interstellar space, because of its high abundance in the Earth's atmosphere. Only indirect searches of the chemically related $\mathrm{HOCO}^{+}$ion have been possible from the ground (Thaddeus et al. 1981; Minh et al. 1988, 1991). The ISO Short Wavelength Spectrometer (SWS) (de Graauw et al. 1996a) opens up the possibility to search for the infraredactive asymmetric stretch and bending modes of gas-phase $\mathrm{CO}_{2}$ around 4.3 and $15.0 \mu \mathrm{m}$ toward bright infrared sources.

The detection of abundant interstellar solid $\mathrm{CO}_{2}$ (de Graauw et al. $1996 \mathrm{~b}$ ) suggests that $\mathrm{CO}_{2}$ could be a particularly sensitive probe of gasgrain chemistry. The observed abundance of solid $\mathrm{CO}_{2}$ with respect to hydrogen of about $\sim(1-5) 10^{-6}$ is a factor of 10 larger than that predicted by cold gas-phase models. Comparison of the observed gas/solid $\mathrm{CO}_{2}$ ratios with those of other species known to be abundant in icy mantles, especially $\mathrm{H}_{2} \mathrm{O}$ and $\mathrm{CO}$, will allow us to address the formation route of $\mathrm{CO}_{2}$.

\section{Observations and analysis}

We searched for gas-phase $\mathrm{CO}_{2}$ in the spectra of four deeply embedded young stellar objects. Mitchell et al. $(1989,1990)$ have measured nearinfrared absorption lines of gas-phase ${ }^{12} \mathrm{CO}$ and ${ }^{13} \mathrm{CO}$ at high spectral resolution from the ground, which indicate the presence of both cold, $T_{\mathrm{kin}}=$ $15-60 \mathrm{~K}$, and warm, $T_{\text {kin }}=120-1000 \mathrm{~K}$, gas along the lines of sight. The same sources are also observed by Helmich et al. (1996, this volume) and van Dishoeck \& Helmich (1996) for gas-phase $\mathrm{H}_{2} \mathrm{O}$ absorption.

Figure 1 shows the observed spectrum between 4.1 and $4.7 \mu \mathrm{m}$ toward GL 2591. It clearly illustrates the difference between the spectrum of a 


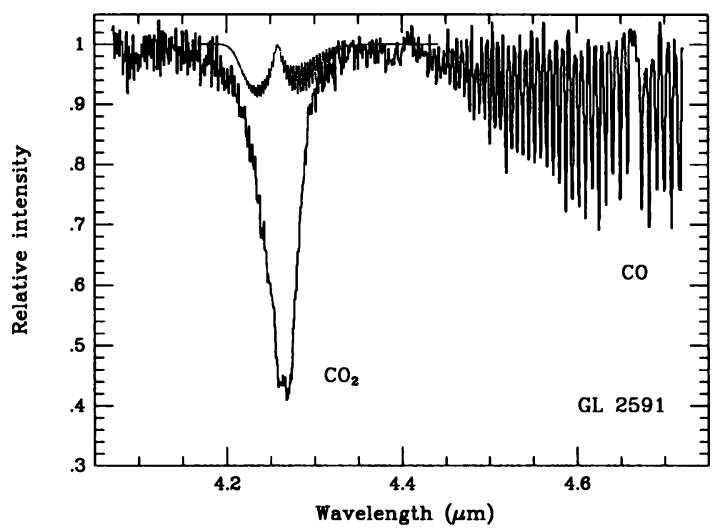

Figure 1. ISO-SWS spectrum toward GL 2591 in the $4.1-4.7 \mu \mathrm{m}$ region. Broad absorption due to the $\nu_{3}$ band of solid $\mathrm{CO}_{2}$ is seen at $4.26 \mu \mathrm{m}$, as well as a forest of $R$ and $P$-branch lines due to the warm, gas-phase CO $v=1-0$ band between 4.4 and $4.7 \mu \mathrm{m}$.

gas-phase and a solid-state species, and indicates a very different chemical behavior for the two species: most of the $\mathrm{CO}$ is in the gas phase, whereas most of the $\mathrm{CO}_{2}$ is in solid form. In order to constrain the amount of gasphase $\mathrm{CO}_{2}$, simulated spectra have been made following Helmich (1996). The adopted Doppler parameters $b$ and excitation temperatures are based on the infrared observations of warm gaseous CO (Mitchell et al. 1990). Figure 1 includes a simulated spectrum (dotted line) of gas-phase $\mathrm{CO}_{2}$ for a column density of $5.10^{16} \mathrm{~cm}^{-2}, b=7.5 \mathrm{~km} \mathrm{~s}^{-1}$, and $T_{\mathrm{ex}}=250 \mathrm{~K}$, which is the maximum column density that could be hidden underneath the solid $\mathrm{CO}_{2}$ feature.

More stringent constraints on the gas-phase $\mathrm{CO}_{2}$ can be deduced from the strong $Q$-branch in the $\nu_{2}$ bending mode. Figure 2 shows the ISOSWS spectra of the four sources around $15 \mu \mathrm{m}$, with model $\mathrm{CO}_{2}$ spectra included. It is seen that in all four sources, a 3-4 $\sigma$ absorption feature is present at the position of the $\mathrm{CO}_{2} \nu_{2} Q$-branch, which tentatively suggests the presence of gas-phase $\mathrm{CO}_{2}$, especially toward GL 2136 and NGC 7538.

The column densities of $\sim 10^{16} \mathrm{~cm}^{-2}$ derived above correspond to gasphase $\mathrm{CO}_{2}$ abundances with respect to total $\mathrm{H}_{2}$ of at most $\sim 10^{-7}$. These abundances are more than an order of magnitude below the limits derived from $\mathrm{HOCO}^{+}$(except for Galactic Center clouds), and a factor of a few lower than predicted by pure gas-phase steady-state models. Typically less than $5 \%$ of the $\mathrm{CO}_{2}$ is in the gas phase, in strong contrast with $\mathrm{CO}$ and $\mathrm{H}_{2} \mathrm{O}$ (see Table 1 ).

The large fraction of $\mathrm{CO}$ in the gas phase is not surprising, because solid CO readily evaporates at dust temperatures above $20 \mathrm{~K}$. The mystery 

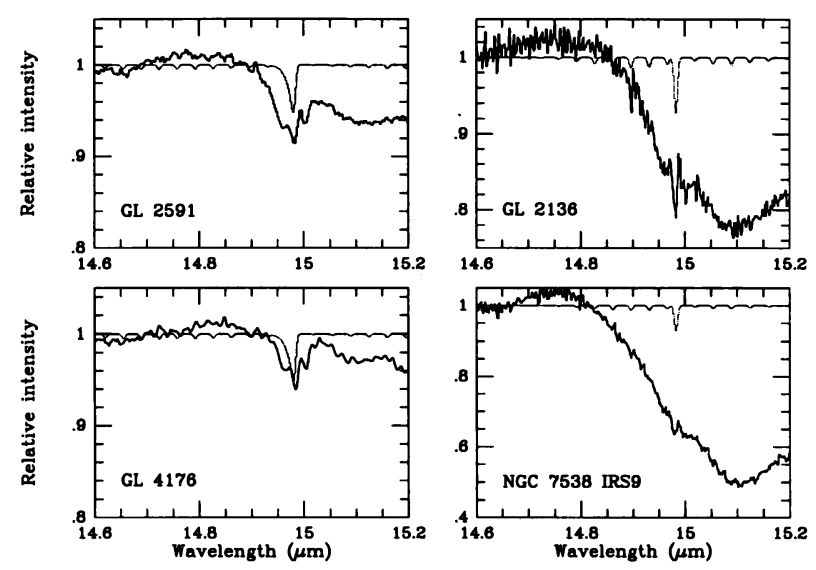

Figure 2. Normalized ISO-SWS grating spectra toward four embedded young stellar objects around $15 \mu \mathrm{m}$. The broad absorption feature starting around $14.8 \mu \mathrm{m}$ is due to solid $\mathrm{CO}_{2}$. The light (dotted) lines indicate the model absorption spectra of gas-phase $\mathrm{CO}_{2}$ for $N\left(\mathrm{CO}_{2}\right)=1.10^{16} \mathrm{~cm}^{-2}$.

TABLE 1. Gas/solid state abundance ratios

\begin{tabular}{lrrr}
\hline Object & $\mathrm{CO}$ & $\mathrm{CO}_{2}$ & $\mathrm{H}_{2} \mathrm{O}$ \\
\hline NGC 7538 IRS9 & 10 & 0.01 & $<0.04$ \\
GL 2136 & 200 & 0.02 & 0.4 \\
GL 2591 & $>400$ & 0.04 & 1.1 \\
GL 4176 & $>400$ & 0.04 & 2.2 \\
\hline
\end{tabular}

is why the gas to solid ratio of $\mathrm{H}_{2} \mathrm{O}$ is so much larger than that of $\mathrm{CO}_{2}$ in three of the sources, because its sublimation temperature of $\sim 45-72 \mathrm{~K}$ is lower than that of $\mathrm{H}_{2} \mathrm{O}(>90 \mathrm{~K})$. Several possible explanations for this problem are discussed by van Dishoeck et al. (1996). The most plausible scenario is that a large fraction of the observed gas-phase $\mathrm{H}_{2} \mathrm{O}$ does not originate only from icy mantles but also from high-temperature gas-phase reactions in shocks or radiatively heated gas.

In summary, the ISO-SWS observations demonstrate that gas-phase $\mathrm{CO}_{2}$ is not abundant in interstellar clouds, in spite of the ubiquitous presence of solid $\mathrm{CO}_{2}$. They also show the potential of ISO to obtain reliable gas to solid state abundance ratios, which should provide insight into the physical and chemical evolution during star formation. 


\section{Acknowledgements}

The authors are grateful to many colleagues who contributed to this work (see full author list in van Dishoeck et al. 1996). They especially thank the SWS Instrument Dedicated Team for their help.

\section{References}

de Graauw, Th., et al., 1996a, A\&A 315, L49

de Graauw, Th., et al., 1996b, A\&A 315, L345

Helmich, F.P., van Dishoeck, E.F., Black, J.H., et al. 1996, A\&A 315, L173

Helmich, F.P., 1996, PhD thesis, University of Leiden

Minh, Y.C., Irvine, W.M., Ziurys, L.M., 1988, ApJ 334, 175

Minh, Y.C., Brewer, M.K., Irvine, W.M., Friberg, P., Johansson, L.E.B., 1991, A\&A 244, 470

Mitchell, G.F., Curry, C., Maillard, J.-P., Allen, M. 1989, ApJ 341, 1020

Mitchell, G.F., Maillard, J.-P., Allen, M., Beer, R., Belcourt, K. 1990, ApJ 363, 554

Thaddeus, P., Guelin, M., Linke, R.A. 1981, ApJ 246, L41

van Dishoeck, E.F., Helmich, F.P., 1996, A\&A 315, L177

van Dishoeck, E.F., Helmich, F.P., de Graauw, Th., et al. 1996, A\&A 315, L349

\section{Discussion}

Buch: Is the assignment of solid $\mathrm{CO}_{2}$ certain?

van Dishoeck: Yes, see the chapter by Tielens \& Whittet in this volume.

Smith: As I understand, the proposed mechanism for formation of $\mathrm{CO}_{2}$ in ices is the reaction of $\mathrm{CO}$ with $\mathrm{O}$ atoms. In the gas phase this spin-forbidden reaction has an appreciable activation energy and there seems little reason to suppose that this will be substantially reduced if the species are simply physisorbed on the surface of interstellar ice grains. Have laboratory experiments confirmed the proposed mechanism for $\mathrm{CO}_{2}$ formation?

van Dishoeck: The formation of $\mathrm{CO}_{2}$ in ices is discussed at several places in this volume, see Tielens \& Whittet and the discussion following Schutte.

Tielens: Comment. Our laboratory experiments show that $\mathrm{O}$ can be stored at $10 \mathrm{~K}$ in a $\mathrm{CO}$ matrix. Upon warm-up, $\mathrm{CO}_{2}$ is immediately formed. Probably the reaction is inhibited by a steric factor and/or small energy barrier. The difference with the earlier experiment by d'Hendecourt et al. likely reflects his much higher $\mathrm{O}_{2} / \mathrm{CO}$ ratio. Under these conditions, atomic $\mathrm{O}$ prefers to react with $\mathrm{O}_{2}$ rather than $\mathrm{CO}$. Beating the drum of grain surface chemistry (again), it should be remembered that on a grain surface an $\mathrm{O}$ atom has a day the time to react with $\mathrm{CO}$ as compared to $10^{-5} \mathrm{~s}$ in the lab. Thus, these experiments show that, depending on the $\mathrm{O} / \mathrm{O}_{2}$ ratio in the accreting gas, $\mathrm{CO}$ can be oxidized to $\mathrm{CO}_{2}$ on a grain surface. 